\title{
Influência do armazenamento na qualidade fisiológica de sementes de soja tratadas com inseticidas
}

\author{
Influence of storage on the physiological quality of soybean seeds treated \\ with insecticides
}

\author{
Gleberson Guillen Piccinin' \\ Alessandro Lucca Braccini2(*) \\ Lilian Gomes de Morais Dan ${ }^{3}$ \\ Gabriel Loli Bazo4 \\ Luiz Henrique da Silva Lima ${ }^{5}$
}

\section{Resumo}

A utilização de inseticidas no tratamento de sementes cresce a cada safra em decorrência do ataque progressivo de diferentes espécies de insetos-pragas. $\mathrm{O}$ objetivo deste trabalho foi avaliar a influência do fipronil e tiametoxam no desempenho fisiológico das sementes de soja sob armazenamento. O delineamento experimental utilizado foi inteiramente casualizado com quatro repetições, distribuído em esquema fatorial (4 x $3 \times 2$ ), utilizou-se quatro cultivares (BMX MAGNA RR, BMX Potência RR, NK 3358 RR e NK 7059 RR), três tratamentos de sementes (fipronil e tiametoxam e uma testemunha sem inseticida), em dois períodos de armazenamento (zero e 180 dias após a colheita). A qualidade fisiológica das sementes foi avaliada pelos testes: germinação (primeira contagem e contagem final), envelhecimento acelerado, frio modificado, comprimento de plântula e raiz. Diante dos resultados obtidos, pode-se inferir que os inseticidas fipronil e tiametoxam influenciam negativamente a qualidade fisiológica das sementes de soja submetidas ao armazenamento por 180 dias. Os inseticidas após o período de armazenamento afeta negativamente o comprimento de raiz.

Palavras-chave: germinação; Glycine max; inseticidas; vigor.

I MSc.; Engenheiro Agrônomo; Doutorando em Agronomia na Universidade Estadual de Maringá, UEM; Endereço:Avenida Colombo, 5790, CEP:87020-900, Maringá, Paraná, Brasil; E-mail: guillen.piccinin@hotmail. com (*) Autor para correspondência.

2 Dr.; Engenheiro Agrônomo; Professor do Departamento de Agronomia da Universidade Estadual de Maringá, UEM; Bolsista de Produtividade em Pesquisa do CNPq; Endereço: Avenida Colombo, 5790, CEP: 87020-900, Maringá, Paraná, Brasil; E-mail: albraccine@uol.com.br (*) Autor para correspondência.

3 MSc.; Engenheira Agrônoma;Doutoranda em Agronomia na Universidade Estadual de Maringá, UEM; Endereço: Avenida Colombo, 5790, CEP: 87020-900, Maringá, Paraná, Brasil; E-mail: liliangmdan@yahoo.com.br

4 Engenheiro Agrônomo; Mestrando em Agronomia na Universidade Estadual de Maringá, UEM; Endereço: Avenida Colombo, 5790, CEP: 87020-900, Maringá, Paraná, Brasil; E-mails: gabriel.loli@hotmail.com; Ihds18@hotmail.com

5 Engenheiro Agrônomo; Mestrando em Genética e Melhoramento na Universidade Estadual de Maringá, UEM; Endereço:Avenida Colombo, 5790, CEP: 87020-900, Maringá, Paraná, Brasil; IhdsI8@hotmail.com

\begin{tabular}{llllll}
\hline Ambiência Guarapuava (PR) & v.9 n.2 & p. 289 - 298 & Maio/Ago. 2013 & ISSN I808 - 025 I
\end{tabular}




\section{Abstract}

The use of insecticide seed treatment grows each season as a result of progressive attack different species of insect pest. The objective of this study was to evaluate the influence of fipronil and thiamethoxam in the physiological performance of soybeans in storage. The experimental design was completely randomized with four replications, distributed a factorial design ( $4 \times 3 \times 2)$, we used four cultivars (BMX Magna RR, BMX Power RR, NK 3358 RR and NK 7059 RR), three seed treatments (fipronil and thiamethoxam insecticide and untreated) in two storage periods (zero and 180 days). The physiological quality of seeds was determined by the tests: germination (first count and final count), accelerated aging, modified cold, seedling length and root. Results can be inferred that the insecticide fitronil and thiamethoxam adversely affect the physiological quality of soy bean seeds subjected to storage for 180 days. The insecticides after the storage period adversely affect the root length.

Key words: germination; Glycine max; insecticides; vigor.

\section{Introdução}

A cultura da soja (Glycine max [L.] Merrill) sofre contínua evolução com elevados investimentos em tecnologia. A prática da utilização do tratamento de sementes com inseticidas no Brasil vem crescendo a cada safra, a fim de proteger as sementes e plântulas contra o ataque de insetos-pragas, o que pode maximizar os resultados da lavoura.

Pragas de solo têm gerado perdas significativas justificando o tratamento preventivo com inseticidas, como a utilização de fipronil e tiametoxam no tratamento de sementes evitando possíveis perdas no estabelecimento inicial $\mathrm{da}$ cultura. No início do desenvolvimento da soja, a germinação uniforme e o crescimento vigoroso são fatores essenciais para manter o máximo potencial produtivo geneticamente contido nas sementes (SILVA, 1998; BAUDET e PESKE, 2007).
É, neste sentido, que o vigor é reconhecido como parâmetro para a caracterização do potencial fisiológico das sementes indicando os lotes com maior ou menor probabilidade de sucesso após a semeadura em campo ou durante o armazenamento (MARCOS FILHO, 2005).

Segundo Tekrony e Egli (1991), o uso de sementes com alto vigor é justificado em todas as culturas, para assegurar adequada população de plantas. Por outro lado, sementes com baixa qualidade fisiológica podem provocar reduções na velocidade de emergência, desuniformidade do "stand", fatores que podem influenciar no rendimento da cultura (SCHUCH et al., 1999; VANZOLINI e CARVALHO, 2002; HÖFS, 2003; KOLCHINSKI et al., 2005).

Embora, haja resultados de pesquisa para o tratamento de sementes de soja com inseticidas, ainda existe carência de informações sobre o armazenamento com sementes tratadas, particularmente, em uma 
safra. Resultados com diversos inseticidas no tratamento de sementes de milho indicaram efeito negativo sobre a germinação das sementes e este efeito intensificouse com o prolongamento do período de armazenamento (BITTENCOURT et al., 2000; FESSEL et al.,2003; BARROS, 2005).

Especificadamente para o inseticida tiametoxam, este demonstrou efeito favorável no crescimento de plântulas de soja. Assim, promoveu a manutenção do vigor, produtividade, área foliar e radicular, apresenta uniformidade na emergência e melhor desenvolvimento inicial (CATANEO et al.,2006; TAVARES et al., 2007; CASTRO, 2008; CLAVIJO, 2008).

Alguns autores relatam que o fipronil promove a maior porcentagem de germinação das sementes Barros et al. (2005), entretanto, Dan et al. (2010a) afirma que é possível ocorrer efeito fitotóxico, em decorrência do aumento do período de armazenamento das sementes tratadas com inseticidas.

Dessa forma, com informações incipientes relacionados ao efeito do tratamento de sementes após o armazenamento, torna-se impreterivelmente necessário a geração de informações sobre a importância da preservação da qualidade fisiológica das sementes durante o armazenamento. $\mathrm{O}$ objetivo do respectivo trabalho foi avaliar a influência do fipronil e tiametoxam no desempenho fisiológico das sementes de soja sob armazenamento.

\section{Material e Métodos}

O trabalho foi desenvolvido no Laboratório de Tecnologia de Sementes do Núcleo de Pesquisas Aplicadas à Agricultura (NUPAGRI) pertencente à Universidade Estadual de Maringá (UEM).
Foram utilizados quatro lotes de sementes das respectivas cultivares de soja: BMX MAGNA RR, BMX Potência RR, NK 3358 RR e NK 7059 RR produzidas na safra agrícola 2010/2011 fornecidas pela Cooperativa Agroindustrial C.Vale.

O teor de água das sementes foi determinado por meio do método de estufa a $105 \pm 3{ }^{\circ} \mathrm{C}$, durante 24 horas (Brasil, 2009), sendo de 10,31, 9,96, 9,20, e 9,76\%, para as cultivares BMX MAGNA RR, BMX Potência RR, NK 3358 RR e NK 7059 RR respectivamente.

O delineamento experimental utilizado foi inteiramente casualizado com quatro repetições, distribuído em esquema fatorial $(4 \times 3 \times 2)$, sendo quatro cultivares, três tratamentos de sementes (fipronil e tiametoxam e uma testemunha sem inseticida), em dois períodos de armazenamento (zero e 180 dias). As sementes, após a colheita foram armazenadas por 60 dias em câmara fria $\left(10{ }^{\circ} \mathrm{C}\right.$ e $45 \%$ de UR do ar).

Para o tratamento das sementes de soja utilizou-se $5 \mathrm{~kg}$ de sementes de cada cultivar, e as dosagens de cada inseticida seguiu a recomendação de registro de 50 g. i. a. $100 \mathrm{~kg}^{-1}$ de sementes para o fipronil (Standak ${ }^{\oplus}$ ) e 70 g. i. a. $100 \mathrm{~kg}^{-1}$ de sementes para o inseticida tiametoxam (Cruiser $700 \mathrm{WS}^{\circledast}$ ).

Por ocasião da aplicação dos inseticidas, a quantidade de cada produto foi diluída em água destilada e veiculada até atingir $150 \mathrm{ml}$ de volume por $50 \mathrm{~kg}$ de sementes, formando uma calda homogênea a fim de proporcionar o total recobrimento das sementes. O tratamento de sementes foi realizado de maneira sequencial em sacos plásticos de $2 \mathrm{~kg}$ de capacidade. Após aplicação da calda com inseticida, o conjunto foi agitado por três minutos visando uniformizar o tratamento sobre 
a massa das sementes. Em seguida, as sementes foram armazenadas em sacos de papel KRAFT ${ }^{\circledast}$, onde uma fração foi submetida à avaliação da qualidade fisiológica das sementes no período zero e a outra fração foi armazenada por 180 dias em condições ambientais não controladas no Laboratório de Tecnologia de Sementes.

A avaliação da qualidade fisiológica das sementes foi realizada por meio dos testes de germinação, envelhecimento acelerado, frio modificado e de comprimento de plântula e raiz. Os referidos testes são descritos a seguir:

Germinação - conduzido utilizandose quatro repetições de 50 sementes, colocadas para germinar entre três folhas de papel-toalha, umedecidas com água deionizada, utilizando-se três vezes a massa do papel seco embebida em água. Foram confeccionados rolos, sendo estes levados para germinador do tipo "Mangelsdorf", regulado para manter a temperatura constante de $25 \pm 1{ }^{\circ} \mathrm{C}$. As avaliações foram realizadas aos cinco (primeira contagem) e oito dias (contagem final), computando-se a porcentagem de plântulas normais, segundo os critérios estabelecidos pelas Regras para Análise de Sementes (BRASIL, 2009). A primeira contagem foi considerada um indicativo do vigor, e a contagem final, a percentagem de total de germinação das sementes.

Envelhecimento acelerado - realizado com quatro sub-amostras de 50 sementes para cada tratamento, distribuídas sobre uma tela fixada no interior de caixas plásticas do tipo "gerbox". No interior de cada caixa, foram adicionados $40 \mathrm{~mL}$ de água destilada para formar uma câmara úmida, e, sobre a tela, colocadas $45 \mathrm{~g}$ de sementes. Em seguida, as caixas foram fechadas, sendo estas levadas a uma câmara de germinação do tipo B.O.D, regulada à temperatura de $41^{\circ} \mathrm{C}$, por 48 horas, conforme as recomendações de (MARCOS FILHO, 1999). Após o período de envelhecimento, as sementes foram submetidas ao teste de germinação, conforme descrito anteriormente, e à avaliação realizada no quinto dia após a semeadura.

Frio modificado - realizado com 200 sementes por tratamento, divididas em quatro sub-amostras de 50 sementes, conforme a metodologia descrita por (BARROS et al., 1999). Como substratos foram utilizados três folhas de papel toalha (germitest), umedecidas com água destilada. Após a semeadura, foram confeccionados rolos, sendo estes envoltos por sacos plásticos, mantendo-se um sistema fechado, permanecendo por um período de cinco dias em câmara de germinação do tipo B.O.D, regulada à temperatura constante de $10{ }^{\circ} \mathrm{C}$. Em seguida, os rolos foram levados para germinador regulado para manter constante a temperatura de $25^{\circ} \mathrm{C}$, durante cinco dias, procedendo-se em seguida a avaliação. Os resultados foram expressos em porcentagem de plântulas normais obtidas na data de avaliação, segundo os critérios adotados para o teste de germinação (BRASIL, 2009).

Comprimento de raiz e plântula - utilizou-se 100 sementes divididas em cinco repetições de 20 sementes de cada tratamento, colocadas para germinar entre três folhas de papel-toalha, umedecidas com água deionizada utilizando-se três vezes a massa do papel seco embebida em água. Sobre o papel-toalha umedecido foi traçada uma linha no terço superior, na direção longitudinal, onde as sementes foram colocadas direcionando-se a micrópila para baixo, após a distribuição 
das sementes confeccionou-se os rolos e posteriormente, levados para germinador do tipo "Mangelsdorf", regulado para manter a temperatura constante de $25 \pm 1{ }^{\circ} \mathrm{C}$, por cinco dias, seguindo a metodologia descrita por Nakagawa, (1999). O comprimento da raiz primária e das plântulas consideradas normais foi determinado ao final do quinto dia, com o auxílio de régua milimetrada (BRASIL, 2009).

As características avaliadas foram submetidas análise de variância e quando significativas, as médias foram comparadas pelo teste SNK (Student Newman Keuls) a 5\% de probabilidade (RAMALHO et al., 2000). Para o fator período de armazenamento o teste $\mathrm{F}$ é conclusivo de acordo com Banzatto e Kronka (2008).

\section{Resultados e Discussão}

$\mathrm{Na}$ tabela 1, estão apresentados os resultados para primeira contagem (vigor) e contagem final (percentual total de germinação). Os tratamentos com fipronil e tiametoxam mantiveram valores semelhantes aos do tratamento controle (sem tratamento com inseticidas) não sendo observados acréscimos significativos para zero dia de armazenamento. Com isso, tanto para primeira contagem quanto para a contagem final, após o tratamento de sementes com fipronil e tiametoxam não observa-se alteração o potencial das sementes de soja. Inclusive, notase neste caso que os tratamentos com fipronil e tiametoxam para zero dia de armazenamento, alcançaram níveis adequados de germinação, com porcentagem acima de $85 \%$ acima de $80 \%$ que o valor mínimo estabelecido por Brasil (2005). De acordo com Dan (2010a), esse resultado caracteriza a ausência de efeitos danosos na utilização de inseticidas imediatamente após o tratamento das sementes.

Dan et al. (2010b) obtiveram resultados semelhantes que ao tratar sementes de soja com fipronil e tiametoxam não afetou a qualidade fisiológica das sementes de soja logo após o tratamento de sementes. Em contrapartida, na primeira contagem para período de armazenamento de 180 dias, as sementes das cultivares BMX Potência RR, NK 3358 RR e NK 7059 $\mathrm{RR}$ tratadas com inseticidas apresentaram significativamente decréscimos no vigor em relação às sementes sem tratamento.

Para o período de 180 dias, na contagem final observou-se que as sementes das cultivares BMX Magna RR, NK 3358 RR e NK 7059 RR quando tratadas com inseticidas fipronil e tiametoxam não apresentaram acréscimos significativos ( $p>0,05)$, exceto para a cultivar BMX Potência RR que apresentou efeito negativo. Com isso, é provável que após 180 dias de armazenamento, as sementes tratadas com 50 g. i. a. $100 \mathrm{~kg}^{-1}$ de sementes de fipronil e 70 g.i.a. $100 \mathrm{~kg}^{-1}$ de tiametoxam não afeta significativamente a porcentagem de geminação. As sementes com baixa qualidade fisiológica interferem no "stand" da lavoura, podendo reduzir drasticamente o rendimento da cultura da soja.

Segundo Castro e Pereira (2008), isso pode ser explicado pelo fato que o tiametoxam movimenta-se através das células das plantas e ativa várias reações fisiológicas, como a expressão de mecanismos de defesa das plantas contra os fatores exógenos nas sementes de soja. 
Tabela I - Porcentagem de plântulas normais na primeira contagem e contagem final do teste de germinação de sementes de soja, sob tratamento de sementes:sem tratamento (S.T), tratamento com fipronil (T.F) e tratamento com tiametoxam (T.T), entre zero e 180 dias de armazenamento

\begin{tabular}{|c|c|c|c|c|c|c|}
\hline \multirow{3}{*}{ Cultivares } & \multicolumn{6}{|c|}{ Primeira contagem } \\
\hline & \multicolumn{3}{|c|}{ zero dias } & \multicolumn{3}{|c|}{180 dias } \\
\hline & S.T & T.F & T. T & S.T & T.F & T.T \\
\hline BMX Magna RR & $92,00 \mathrm{a}$ & $91,25 \mathrm{a}$ & $91,50 \mathrm{a}$ & $80,00 \mathrm{a}$ & $76,25 \mathrm{a}$ & $78,50 \mathrm{a}$ \\
\hline BMX Potência RR & $89,75 \mathrm{a}$ & $90,25 \mathrm{a}$ & $90,25 \mathrm{a}$ & $82,50 \mathrm{a}$ & $68,75 \mathrm{~b}$ & $68,25 \mathrm{~b}$ \\
\hline NK 3358 RR & $89,00 \mathrm{a}$ & $89,00 \mathrm{a}$ & $89,75 \mathrm{a}$ & $83,00 \mathrm{a}$ & $72,50 \mathrm{c}$ & $77,00 \mathrm{~b}$ \\
\hline NK 7059 RR & $90,00 \mathrm{a}$ & $86,00 \mathrm{a}$ & $85,00 \mathrm{a}$ & $81,75 \mathrm{a}$ & $74,25 \mathrm{~b}$ & $76,75 \mathrm{~b}$ \\
\hline C.V $(\%)$ & 3,66 & & & & & \\
\hline \multirow{3}{*}{ Cultivares } & \multicolumn{6}{|c|}{ Contagem final } \\
\hline & \multicolumn{3}{|c|}{ zero dias } & \multicolumn{3}{|c|}{180 dias } \\
\hline & S.T & T.F & T.T & S.T & T. F & T. T \\
\hline BMX Magna RR & $94,75 \mathrm{a}$ & $93,25 \mathrm{a}$ & $94,25 \mathrm{a}$ & $85,50 \mathrm{a}$ & $79,25 \mathrm{a}$ & $80,75 \mathrm{a}$ \\
\hline BMX Potência RR & $93,00 \mathrm{a}$ & $92,25 \mathrm{a}$ & $92,25 \mathrm{a}$ & $91,25 \mathrm{a}$ & $73,00 \mathrm{~b}$ & $71,25 \mathrm{~b}$ \\
\hline NK 3358 RR & $93,50 \mathrm{a}$ & $92,50 \mathrm{a}$ & $92,75 \mathrm{a}$ & $85,50 \mathrm{a}$ & 74,75 a & $78,50 \mathrm{a}$ \\
\hline NK 7059 RR & $91,75 \mathrm{a}$ & $91,25 \mathrm{a}$ & $78,50 \mathrm{a}$ & $84,25 \mathrm{a}$ & $76,50 \mathrm{a}$ & $79,00 \mathrm{a}$ \\
\hline C.V $(\%)$ & 9,58 & & & & & \\
\hline
\end{tabular}

Fonte:Autores (20I2).

Nota: Médias seguidas da mesma letra minúscula na linha, não diferem entre si, pelo teste SNK (Student Newman Keuls) a $5 \%$ de probabilidade.

Tabela 2 - Resultados médios em porcentagem de plântulas normais no teste de envelhecimento acelerado e frio modificado, sob tratamento de sementes de soja: sem tratamento (S.T), tratamento com fipronil (T.F) e tratamento com tiametoxam (T.T), entre zero e 180 dias de armazenamento

\begin{tabular}{|c|c|c|c|c|c|c|}
\hline \multirow{3}{*}{ Cultivares } & \multicolumn{6}{|c|}{ Envelhecimento acelerado } \\
\hline & \multicolumn{3}{|c|}{ zero dias } & \multicolumn{3}{|c|}{180 dias } \\
\hline & S.T & T.F & T.T & S.T & T.F & T.T \\
\hline BMX Magna RR & $90,00 \mathrm{a}$ & $89,00 \mathrm{a}$ & $89,25 \mathrm{a}$ & $82,00 \mathrm{a}$ & $76,50 \mathrm{~b}$ & $77,00 \mathrm{~b}$ \\
\hline BMX Potência RR & $89,25 \mathrm{a}$ & $91,25 \mathrm{a}$ & $88,00 \mathrm{a}$ & $83,25 \mathrm{a}$ & $72,75 \mathrm{~b}$ & $72,00 \mathrm{~b}$ \\
\hline NK 3358 RR & $83,00 \mathrm{ab}$ & $80,00 \mathrm{~b}$ & $87,00 \mathrm{a}$ & $79,25 \mathrm{a}$ & $72,50 \mathrm{~b}$ & $75,50 \mathrm{ab}$ \\
\hline NK 7059 RR & $86,25 \mathrm{a}$ & $81,50 \mathrm{a}$ & $82,25 \mathrm{a}$ & $84,50 \mathrm{a}$ & $71,25 \mathrm{~b}$ & $74,00 \mathrm{~b}$ \\
\hline C.V (\%) & 3,59 & & & & & \\
\hline \multirow{3}{*}{ Cultivares } & \multicolumn{6}{|c|}{ Frio modificado } \\
\hline & \multicolumn{3}{|c|}{ zero dias } & \multicolumn{3}{|c|}{180 dias } \\
\hline & S.T & T. F & T.T & S.T & T. F & T.T \\
\hline BMX Magna RR & $89,25 \mathrm{a}$ & $89,25 \mathrm{a}$ & $90,75 \mathrm{a}$ & $84,25 \mathrm{a}$ & $76,75 \mathrm{~b}$ & $78,25 \mathrm{~b}$ \\
\hline BMX Potência RR & 86,75 a & $84,25 \mathrm{a}$ & $85,25 \mathrm{a}$ & $81,25 \mathrm{a}$ & $74,50 \mathrm{~b}$ & $75,00 \mathrm{~b}$ \\
\hline NK 3358 RR & $89,75 \mathrm{a}$ & $88,50 \mathrm{a}$ & $89,75 \mathrm{a}$ & $81,00 \mathrm{a}$ & $75,75 \mathrm{a}$ & $75,25 \mathrm{a}$ \\
\hline NK 7059 RR & $91,50 \mathrm{a}$ & $89,75 \mathrm{a}$ & $89,00 \mathrm{a}$ & $86,25 \mathrm{a}$ & $77,00 \mathrm{~b}$ & $78,25 \mathrm{~b}$ \\
\hline C.V (\%) & 4,53 & & & & & \\
\hline
\end{tabular}

Fonte:Autores (20I2).

Nota: Médias seguidas da mesma letra minúscula na linha, não diferem entre si, pelo teste SNK (Student Newman Keuls) a $5 \%$ de probabilidade. 
Tabela 3 - Resultados médios de comprimento de plântula e raiz em centímetros, sob tratamento de sementes de soja: sem tratamento (S.T), tratamento com fipronil (T.F) e tratamento com tiametoxam (T.T), entre zero e 180 dias de armazenamento

\begin{tabular}{|c|c|c|c|c|c|c|}
\hline \multirow{3}{*}{ Cultivares } & \multicolumn{6}{|c|}{ Comprimento de plântula } \\
\hline & \multicolumn{3}{|c|}{ zero dias } & \multicolumn{3}{|c|}{180 dias } \\
\hline & S.T & T.F & T. T & S.T & T. F & T.T \\
\hline BMX Magna RR & $9,56 \mathrm{a}$ & $9,53 \mathrm{a}$ & $10,53 \mathrm{a}$ & $7,78 \mathrm{a}$ & $6,99 \mathrm{a}$ & $5,73 \mathrm{a}$ \\
\hline BMX Potência RR & $8,59 \mathrm{a}$ & $7,54 \mathrm{a}$ & $9,54 \mathrm{a}$ & $7,59 \mathrm{a}$ & $5,50 \mathrm{a}$ & $5,29 \mathrm{a}$ \\
\hline NK 3358 RR & $8,62 \mathrm{a}$ & $8,14 \mathrm{a}$ & $8,84 \mathrm{a}$ & $6,58 \mathrm{a}$ & $5,20 \mathrm{a}$ & $5,38 \mathrm{a}$ \\
\hline NK 7059 RR & $8,45 \mathrm{a}$ & $7,89 \mathrm{a}$ & $9,56 \mathrm{a}$ & $6,65 \mathrm{a}$ & $6,49 \mathrm{a}$ & $6,81 \mathrm{a}$ \\
\hline C.V (\%) & 21,55 & & & & & \\
\hline \multirow{3}{*}{ Cultivares } & \multicolumn{6}{|c|}{ Comprimento de raiz } \\
\hline & \multicolumn{3}{|c|}{ zero dias } & \multicolumn{3}{|c|}{180 dias } \\
\hline & S.T & T.F & T.T & S.T & T.F & T.T \\
\hline BMX Magna RR & $18,36 \mathrm{a}$ & $17,30 \mathrm{a}$ & $16,60 \mathrm{a}$ & $15,96 \mathrm{a}$ & $13,82 \mathrm{a}$ & $11,06 \mathrm{a}$ \\
\hline BMX Potência RR & $16,47 \mathrm{a}$ & $15,89 \mathrm{a}$ & $15,80 \mathrm{a}$ & $14,47 \mathrm{a}$ & $12,35 \mathrm{ab}$ & $10,35 \mathrm{~b}$ \\
\hline NK 3358 RR & $17,96 \mathrm{a}$ & $15,86 \mathrm{a}$ & $15,78 \mathrm{a}$ & $14,58 \mathrm{a}$ & $11,32 \mathrm{~b}$ & $12,32 \mathrm{ab}$ \\
\hline NK 7059 RR & $17,31 \mathrm{a}$ & $16,47 \mathrm{a}$ & $16,27 \mathrm{a}$ & $15,66 \mathrm{a}$ & $10,75 \mathrm{~b}$ & $11,35 \mathrm{~b}$ \\
\hline C.V $(\%)$ & 13,05 & & & & & \\
\hline
\end{tabular}

Fonte:Autores (20I2).

Nota: Médias seguidas da mesma letra minúscula na linha, não diferem entre si, pelo teste SNK (Student Newman Keuls) a 5\% de probabilidade.

No teste de envelhecimento acelerado (Tabela 2), para o tempo de zero dia de armazenamento, nota-se que apenas a cultivar NK 3358 RR apresentou desempenho significativamente inferior a $5 \%$ de probabilidade para o tratamento de sementes com fipronil quando comparado com os demais tratamentos. Para o período de armazenamento de 180 dias, todas as cultivares tratadas com o inseticida fipronil apresentaram desempenho significativamente inferior em relação as não tratadas.

A ausência de significância quando realizou o tratamento das sementes com inseticida fipronil contrasta com os resultados obtidos por Horii e Shetty (2007) e Vanin et al. (2011) que trabalharam com a cultura do sorgo, encontraram efeitos benéficos no armazenamento das sementes, promovendo maior emergência de plântulas, quando foram tratadas com o inseticida fipronil e com a mistura tiametoxam + fipronil.

Assim, as sementes tratadas com tiametoxam também apresentaram redução na porcentagem de plântulas normais, exceto para cultivar NK 3358 RR. Por outro lado, Almeida et al. (2011), verificaram para a cultura do arroz incremento na qualidade fisiológica das sementes quando realizou-se o tratamento com tiametoxam.

Pode-se observar que os dados referentes ao período de armazenamento de 180 dias revelam que os inseticidas fipronil e tiametoxam diminuíram significativamente $(p<0,05)$ o vigor das sementes das cultivares BMX Magna RR, BMX Potência RR e NK 7059 RR. No entanto, os resultados nos indicam que o tratamento de sementes 
após o período de armazenamento por 180 dias apresenta uma tendência em afetar significativamente a qualidade fisiológica das sementes de soja.

Os resultados obtidos para o comprimento de plântula e de raiz encontramse na tabela 3. Para o comprimento de plântula, não houve influencia significativa dos tratamentos. No entanto, para o comprimento de raiz, o tratamento com o fipronil no período de 180 dias de armazenamento apresentou redução significativa no comprimento de raiz das cultivares NK 3358 RR e NK 7059 RR. Já para as cultivares BMX Potência RR e NK 7059 RR o tratamento com tiametoxam afetou negativamente o comprimento de raiz. Esses resultados condizem com os obtidos por Dan et al. (2010a) que obtiveram efeitos negativos no comprimento das raízes quando as sementes de soja foram tratadas com fipronil, acefato e tiametoxam.
Os resultados nos indicam que tratamento de sementes com fipronil e tiametoxam após 180 dias, pode provocar alterações degenerativas no metabolismo, bem como desencadear o processo de desestruturação das membranas celulares das sementes obtendo no qual conferiria em uma queda no vigor das sementes.

\section{Conclusões}

O tratamento de sementes com os inseticidas fipronil e tiametoxam prejudica a qualidade fisiológica das sementes quando submetidas ao armazenamento por 180 dias em condições normais de armazenamento.

O armazenamento das sementes por 180 dias tratadas com fipronil e tiametoxam influencia negativamente o comprimento de raízes.

\section{Referências}

ALMEIDA, A. S.; CARVALHO, I.; DEUNER, C.; ANGELA, M.; TILLMANN, A.; VILLELA, F. A. Bioativador no desempenho fisiológico de sementes de arroz. Revista Brasileira de Sementes, Londrina, v. 33, n. 3, p. 501 - 510, 2011.

BANZATTO,D. A.; KRONKA, S. N. Experimentação agrícola. 4. ed. Jaboticabal: FUNEP, 2008. 237p.

BARROS, A. S. R.; DIAS, M. C. L. L.; CÍCERO, S. M.; KRZYZANOWSKI, F. C. Teste de frio. In: KRZYZANOWSKI, F. C.; VIEIRA, R. D.; FANÇA NETO, J. B. (Ed.). Vigor de sementes: conceitos e testes. Londrina: ABRATES, 1999. p.5.1-5.13

BARROS, R. G.; BARRIGOSSI, J. A. F.; COSTA, J. L. S.; Efeito do armazenamento na compatibilidade de fungicidas e inseticidas, associados ou não a um polímero no tratamento de sementes de feijão. Bragantia, Campinas, v.64, n.3, p.459-465, 2005.

BAUDET, L.; PESKE, F. Aumentando o desempenho das sementes. Seed News, v.9, n.5, p.22-24, 2007. 
BITTENCOURT, S. R. M.; FERNANDES, M. A.; RIBEIRO, M. C. R.; VIEIRA, R. D. Desempenho de sementes de milho tratadas com inseticidas sistêmicos. Revista Brasileira de Sementes, v.22, n.2, p.86-93, 2000.

BRASIL. Ministério de Agricultura, Pecuária Abastecimento. Instrução Normativa n.25, de 16 de dezembro de 2005. Diário Oficial [da] República Federativa do Brasil, Poder Executivo, Brasília, DF, 16 dez. 2005.p.18.

BRASIL. Ministério da Agricultura, Pecuária e Abastecimento. Regras para análise de sementes. Ministério da Agricultura, Pecuária e Abastecimento. Secretaria de Defesa Agropecuária. Brasília, DF: Mapa/ACS, 2009. 395p.

CASTRO, P. R. C.; PEREIRA, M. A. Bioativadores na agricultura. In: GAZZONI, D. L. (Coord.). Tiametoxam: uma revolução na agricultura brasileira. Petrópolis: Vozes, 2008. p. 115-122.

CATANEO, A. C.; ANDRÉO, Y.; SEIFFERT, M.; BÚFALO, J.; FERREIRA, L. C. Ação do inseticida Cruiser sobre a germinação do soja em condições de estresse. In: CONGRESSO BRASILEIRO DE SOJA, 4., 2006, Londrina. Resumos... Londrina: Embrapa Soja, 2006.p.26.

CLAVIJO, J. Tiametoxam: um nuevo concepto em vigor y productividad. Bogotá: Co; Ed. Arte Litográfico, 2008. 196 p.

DAN, L. G.M.; DAN, H. A.; BARROSO, A. L.L.; BRACCINI, A. L. Qualidade fisiológica de sementes de soja tratadas com inseticidas sob efeito do armazenamento. Revista Brasileira de Sementes, v. 32, n. 2 p. 131-139, 2010a.

DAN, L. G. M.; DAN, H. A.; BARROSO, A. L. L.; CÂMARA, A.C. F.; GUADANIN, E. C. Efeito de diferentes inseticidas sobre a qualidade fisiológica de sementes de soja. Global Science and Technology, v. 3, n. 1, p. 50-57, 2010b.

FESSEL, S.A.; MENDONCA, E. A.F.; CARVALHO, R.V.Effect of chemical treatment on corn seeds conservation during storage. Revista Brasileira de Sementes, v.25, n.1, p.25-28, 2003.

HÖFS, A.; SCHUCH, L. O. B.; PESKE, S. T.; BARROS, A. C. S. A. Efeito da qualidade fisiológica das sementes e da densidade de semeadura sobre o rendimento de grãos e qualidade industrial em arroz. Revista Brasileira de Sementes, Pelotas, v.26, n.2, p.55-62, 2004.

HORII, P.M.; SHETTY, K. Enhancement of seed vigour following insecticide and phenolic elicitor treatment. Bioresource Technology, v. 98, p.623-632, 2007.

KOLCHINSKI, E. M.; SCHUCH, L. O. B.; PESKE, S. T. Vigor de sementes e competição intra-específica em soja. Ciência Rural, Santa Maria, v.35, n.6, p.1248-1256, 2005.

MARCOS FILHO, J. Teste de envelhecimento acelerado. In: KRZYZANOWSKI, F. C.; VIEIRA, R. D.; FRANÇA NETO, J. B. (Ed.). Vigor de sementes: conceitos e testes. Londrina: ABRATES, 1999. cap.3. p.1-24. 
MARCOS FILHO, J. Fisiologia de sementes de plantas cultivadas. Piracicaba: Fealq, $2005.425 \mathrm{p}$.

NAKAGAWA, J. Testes de vigor baseados no desempenho das plântulas. In: KRZYZANOWSKI, F. C.; VIEIRA, R. D.; FRANÇA NETO, J. B. (Ed.). Vigor de sementes: conceitos e testes. Londrina: ABRATES, 1999. cap.3. p.1-24.

RAMALHO, M. A. P.; FERREIRA, D. F.; OLIVEIRA, A. C. de. A experimentação em genética e melhoramento de plantas. Lavras: UFLA, 2000. 326 p.

ROYALTY, R. N.; LONG, N. D.; PILATO, M. T.; HAMON, N. M. Plant growth promotion using 3-cyano 1-phenylpirazoles such as fipronil. 1996. United States Patent. Disponível em: < http://www.freepatentsonline.com/5585329.pdf>. Acesso em: 10 mar. 2011.

SCHUCH, L.O. B.; NEDEL, J. L.; ASSIS, F.N.; MAIA, M. S. Crescimento em laboratório de plântulas de aveia-preta (Avena strigosa Schreb.) em função do vigor das sementes. Revista Brasileira de Sementes, v.21, n.1, p.229-234, 1999.

SILVA, M. T. B. Inseticidas na proteção de sementes e plantas. Seed News, v.2, n.5, p.2627, 1998.

TEKRONY, D. M.; EGLI, D. B. Relationship of seed vigor to crop yield: A review. Crop Science, v.31, p.816-822, 1991.

TAVARES, S.; CASTRO, P. R. C.; RIBEIRO, R. V.; ARAMAKI,P.H. Avaliação dos efeitos fisiológicos do tiametoxam no tratamento de sementes de soja. Revista de Agricultura, v. 82, n. 1, p. 47-54, 2007.

VANIN, A.; SILVA, A. G.; FERNANDES, C. P. C.; FERREIRA, W. S.; RATTES, J. F. Tratamento de sementes de sorgo com inseticidas. Revista Brasileira de Sementes, v. 33, n. 2, p. 299 - 309, 2011.

VANZOLINI, S.; CARVALHO, N. M. Efeito do vigor de sementes de soja sobre o seu desempenho em campo. Revista Brasileira de Sementes, v.24, n.1, p.33-41, 2002. 